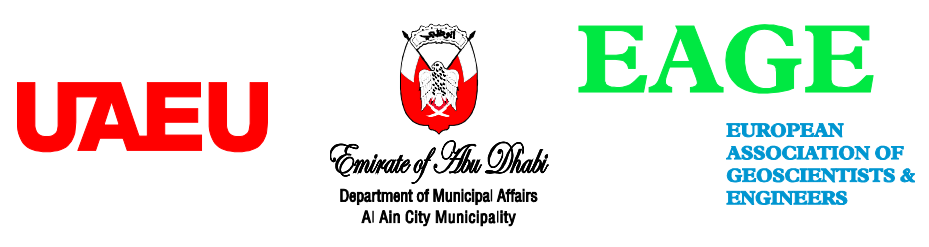

\title{
Introduction
}

Especially in urban areas, tunneling is the method of choice to built new pathways to improve the infrastructure, for e.g. rail tracks, roads or power cables. In this context, safety threads are not limited to the tunneling construction itself but can occur years later. Cavities or fracture zones that can be weakened by the tunneling are a serious risk, both for the stability and integrity of the tunnel tube and buildings on the surface. A collapse of a cavity can result in sudden load peaks possibly overpowering the stability of the tunnel casing and subsidence damage to buildings and buried gas pipelines (Fig. 1). Seismic tomography is a useful tool to detect such anomalies in the vicinity of the tunnel tube while the tunneling progresses or after completion. In order to do so, seismic receivers can be placed at the tunnel wall or at anchors behind the tunnel wall. The seismic wave field is excited by a hammer blow applied to the tunnel wall. Basis for such a tomography is a profound understanding of the seismic wave propagation in the complex surrounding of a tunnel which can be gained from seismic modeling. We, therefore, investigate the influence of the excavation damaged zone (EDZ) that is usually present as a side effect of the tunneling and the topography of the tunnel wall. Both features will significantly effect the seismic waves excited at the tunnel wall. The modeling is done by the parallel elastic 3-D finite difference (FD) modeling code SOFI3D using a Cartesian coordinate system [1]. Later, we insert an anomaly close to the tunnel wall. This paper will now primary focus on the realistic description of a tunnel example and the accurate modeling of seismic waves with respect to this model.

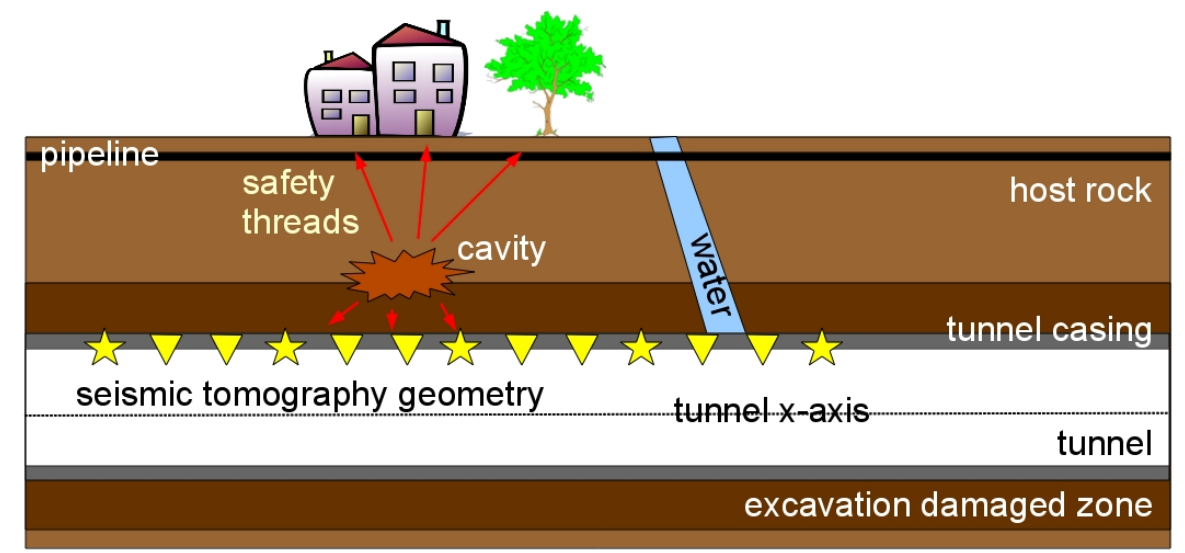

Fig. 1 Schematic side view of a tunnel construction after completion. Unknown cavities or water bearing fractures in the vicinity of the tunnel construction can cause safety threads both affecting the stability of the tunnel and the surface. Yellow stars (sources) and triangles (receiver) illustrate a seismic tomography geometry for the detection of such anomalies.

\section{FD modeling}

The basis for our studies is a 3-D random media volume model which includes random variations in elastic properties (density, $\mathrm{P}$ - and S-wave velocity) and structures at different length scales. In this way, we simulate a heterogeneous and irregular structured rock formation with elastic property variations of up to $5 \%$. The seismic formation properties base on a typical hard rock $(v p=5800 \mathrm{~m} / \mathrm{s}, \mathrm{vs}=3100 \mathrm{~m} / \mathrm{s}$, density=2100 $\mathrm{kg} / \mathrm{m} 3)$. Even though tunneling in urban areas is mostly performed in soft rocks (sediments) below the water table we have chosen the hard rock example to be able to compare our synthetic seismograms with existing field data acquired in crystalline host rock. 


\section{UAEU \\ Emirate of Oflw ODabi \\ Department of Munlclipal Affalrs
Al Ain City Municipality

In our model, the excavated material is replaced with vacuum $(\mathrm{vp}=\mathrm{vs}=0 \mathrm{~m} / \mathrm{s})$. Also, the tunnel is surrounded by a simulated excavation damaged zone (EDZ). In agreement with common assumptions on the dimension of the EDZ ([2], [3]), the modeled EDZ extends twice the tunnel radius into the surrounding formation and is approximated by a seismic velocity and density gradient. We applied a linear decrease of up to $20 \%$ of the original random media formation velocities and density toward the tunnel surface (Fig. 2a). This causes the TS-wave to exhibit medium related dispersion. A point force source is applied to one side of the tunnel wall and various 3-component receiver lines record the wave field along the tunnel wall. Note that due to the relative gradient applied to the random media model, the absolute gradient varies with the azimuth, i.e. the EDZ is not radial symmetric with respect to the tunnel $\mathrm{x}$-axis (Fig. 2b).

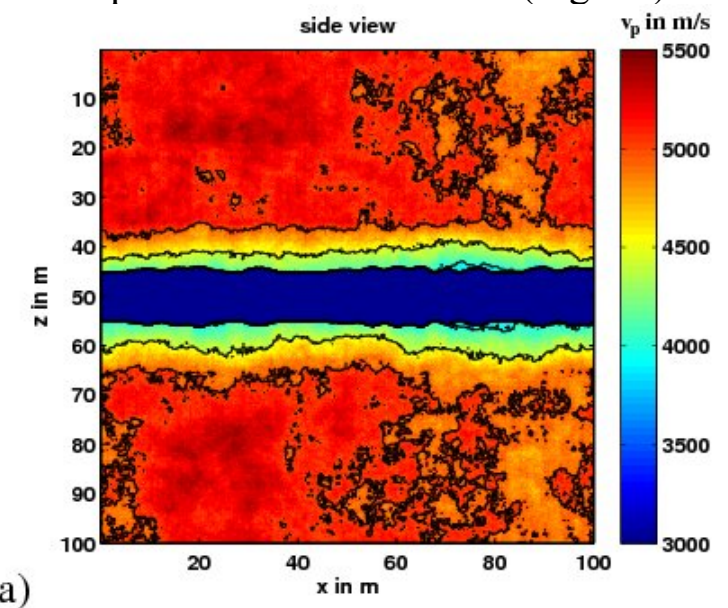

b)

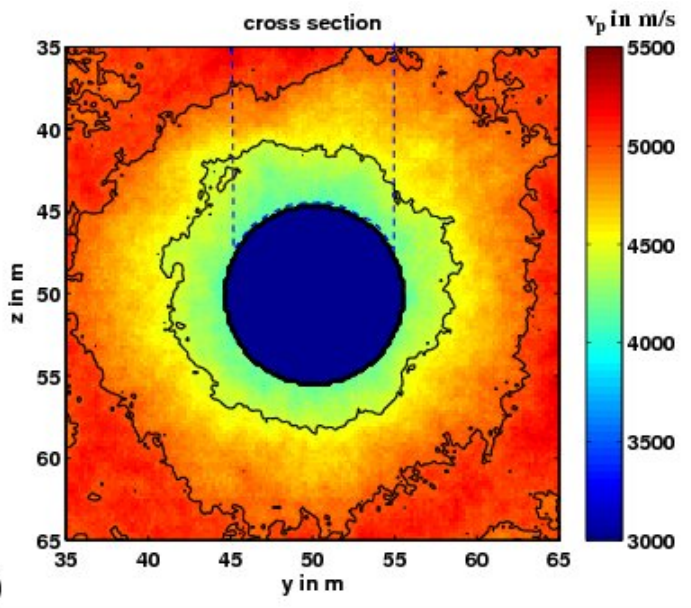

Fig. 2 Slices within the 3-D P-wave velocity volume used for the seismic wave modeling: a) Vertical section slicing the tunnel axis. b) Zoomed section of the tunnel cross section at a distance of $x=50 \mathrm{~m}$. The blue dashed line outline the low velocity anomaly as a target for the tomography. For visibility, the P-wave velocities are clipped to the lower boundary of $3000 \mathrm{~m} / \mathrm{s}$ and contour lines of the velocity model are included (black lines).

For the accurate modeling of body-waves, used for a travel time tomography, as well as surface waves, also used for a full wave form tomography (FWT), the spatial discretization dh of the FD model grid is critical. A previous study has shown that surface waves have to be sampled by at least 30 grid points per typical surface-wave wavelength [4]. In our modeling example, the tunnel surface-wave (TS-wave) wavelength is about $6.7 \mathrm{~m}$ (source center frequency is $300 \mathrm{~Hz}$ and the minimum TS-wave velocity is about $2000 \mathrm{~m} / \mathrm{s}$ ). The grid spacing (distance between two neighboring grid points) is therefore chosen to be $\mathrm{dh}=0.2 \mathrm{~m}$. To give an example, a grid spacing of $\mathrm{dh}=0.2 \mathrm{~m}$ means that, due to the Cartesian coordinate system, a circular break out of $1.0 \mathrm{~m}$ depth is discretized by 5 rectangular steps. The tunnel wall in our model is represented by a smooth spline curve with random sampling points. We allow for a maximum deviation of $1.0 \mathrm{~m}$ from the original tunnel radius of $5.0 \mathrm{~m}$. Note that this variation is only applied to the the tunnel wall in x-direction (Figure 2a), i.e. the overall tunnel diameter is changing with respect to the $\mathrm{x}$-axis. There is no azimuthal variation in the cross section, i.e. the tunnel cross section is always circular (Fig. 2b).

In order to avoid effects of the discretization of the tunnel wall, a single computation has been performed on an interpolated grid with a grid spacing of $\mathrm{dh}=0.1 \mathrm{~m}$. While keeping in mind, that due to the interpolation the models at $\mathrm{dh}=0.1 \mathrm{~m}$ and $\mathrm{dh}=0.2 \mathrm{~m}$ are slightly different, the seismogram are almost the same. Therefore, for the realistic modeling of the 


\section{UAEU

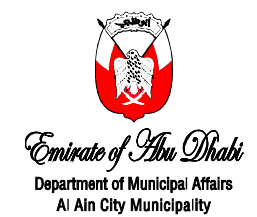

tunnel wall topography the grid spacing $\mathrm{dh}=0.2 \mathrm{~m}$ is ideal. A shorter spacing results in larger computation time, a larger spacing will limit the smallest structure size within the volume.

Each single wave simulation on a grid with 125 million grid points is performed at the Juropa supercomputer of the Juelich Supercomputer Centre (JSC). A run with 80 CPUs takes about 4 hours. For the seismic tomography we plan to simulate 20 shot positions and wave modelings, respectively.

\section{Preliminary results}

To illustrate the different seismic response, we plot common source seismogram sections according to a random media model with and without EDZ and tunnel wall topography in Fig. 3. Additionally, a rectangular low velocity body has been inserted along the receiver line in the distance range $\mathrm{x}=50-55 \mathrm{~m}$ (Fig. $3 \mathrm{~b}$, blue dashed outline). The velocity decrease towards this anomaly is less then $20 \%$ of the original host rock parameters. This anomaly can be treated as a simplification of a water bearing zone, where the water content decreases the elastic properties. The most prominent wave type in Fig. 3a is the tunnel surface-wave (TSwave) which is typical for a force source excitation perpendicular to the tunnel wall [5]. The TS-wave that circulates the tunnel tube is also visible, direct P-wave arrivals are weak by comparison. Due to the fairly large TS-wave wavelength most of the internal structure within the random media does not effect the wave propagation. Arrivals of all three wave types can be seen in Fig. $3 b$, too. In addition, a lot of scattering events can be observed, especially at the interface of the low velocity anomaly. Even though the EDZ gradient is significant, the TS-wave shows dispersion effects only at larger offsets than $40 \mathrm{~m}$ (distance of $67 \mathrm{~m}$ ).

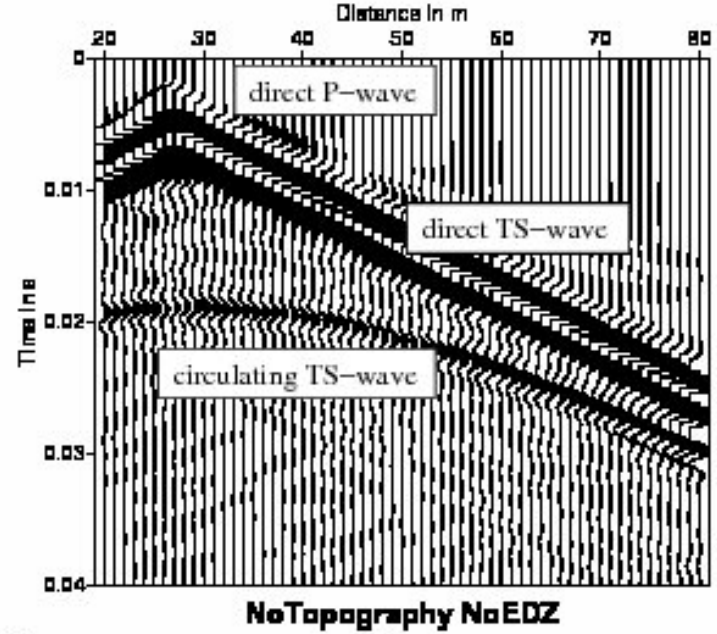

a)

Fig. 3 Seismogram sections (vertical component, perpendicular to the tunnel wall) with a vertical force source position at $x=27 \mathrm{~m}$ and $2 \mathrm{~m}$ behind the tunnel wall. according to a) the random media model without EDZ and tunnel wall topography and b) the random media model with EDZ, tunnel wall topography and a low velocity anomaly within the distance range 50-55 $\mathrm{m}$ (blue dashed lines).

\section{Conclusions and outlook}

On the basis of a 3-D random media with small and large scale variations in the elastic parameters, we have created a realistic tunnel model that accounts for typical features encountered during tunnel construction. First the tunnel wall exhibits a tunnel wall 


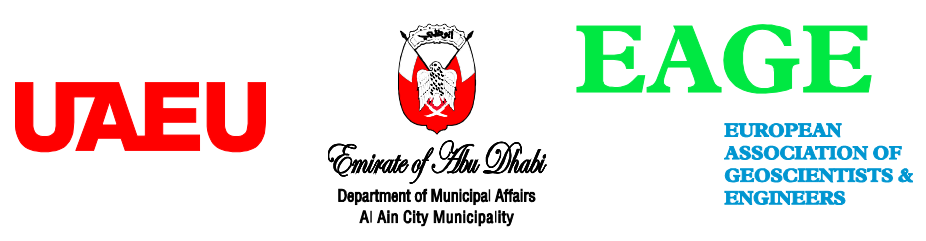

topography that acts as an implicit free surface boundary condition, i.e. the tunnel diameter varies with respect to the tunnel $\mathrm{x}$-axis. Numerical efforts and tests have been made to ensure the accuracy of the seismic response independently of the spatial discretization of the model grid. A more realistic tunnel wall topography would of course include azimuthal variations in the radius of the tunnel, i.e. the tunnel cross section is not circular any more. Second, we applied a gradient in the elastic parameters to simulate the excavation damaged zone (EDZ) which usually occurs as a result of the mechanical load during the tunnel construction. Both features significantly influence the seismic wave propagation. Finally, we inserted a rectangular low velocity anomaly close to the tunnel wall which can be treated as a water bearing zone. We plan to reduce or vary the size and shape of the body to be able to perform a resolution analysis for different kinds of anomalies (e.g. thin layers for fractures, small evacuated bodies for cavities).

By the help of 3-D elastic finite difference modeling using SOFI3D [1], we simulated and studied the seismic response according to the tunnel model. This is the intermediate step towards a seismic tomography to detect anomalies such as cavities or water bearing fractures in the vicinity of the tunnel wall. We plan to first employ a standard travel time tomography that interprets the first arrivals of the P-wave. Later, we can use the results as a starting model for a full waveform tomography (FWT) that exploits the full content of the recorded waveforms (body waves and surface waves at once). This method promises higher resolved images of the tunnel surrounding. Overall goal is the the adaption of existing tomography tools for a robust and reliable detection of possible safety threads behind the tunnel wall.

\section{Acknowledgements}

This study has been performed within the project SOUND which is part of the R\&D-program GEOTECHNOLOGIEN. The project SOUND is funded by the German Ministry of Education and Research (BMBF), Grant 03G0738C.

\section{References}

[1] T. Bohlen, "Parallel 3-D Viscoelastic Finite-Difference Seismic Modelling, Computers \& Geosciences, vol. 28, no. 8, 887-899, 2002, www.gpi.kit.edu/SOFI3D.php.

[2] K. Schuster, H.-J. Alheid, and D. Boddener, "Seismic investigation of the Excavation damaged zone in Opalinus Clay", Engineering Geology, vol. 61, no. 2, pp. 189- 197, 2001.

[3] G. Borm, R. Giese, P. Otto, F. Amberg and T. Dickmann, "Integrated Seismic Imaging System for geological prediction during tunnel construction", ISRM 2003 - Technology roadmap for rock mechanics, South African Institute of Mining and Metallurgy, 2003.

[4] T. Bohlen and E.H. Saenger, "Accuracy of heterogeneous staggered-grid finite-difference modeling of Rayleigh waves", Geophysics, vol. 71, no. 4, T109-T115, 2006

[5] S. Jetschny, D. De Nil and T. Bohlen, "On the propagation characteristics of tunnel surfacewaves for seismic prediction”, Geophysical Prospecting, vol. 2, no. 58, 245--256, 2010 